\title{
Time Shifted Pilot Signal Transmission With Pilot Hopping To Improve The Uplink Performance of Massive MIMO System For Next Generation Network
}

\author{
Amrita Ruperee $^{1^{*}}$, and Shikha Nema ${ }^{2}$ \\ ${ }^{1}$ Department of Electronics and Communication Engineering, \\ S.N.D.T. University, Mumbai-400015 - INDIA \\ [e-mail: amritaruperee@gmail.com] \\ ${ }^{2}$ Department of Electronics and Communication Engineering, \\ S.N.D.T. University, Mumbai-400015 - INDIA \\ [e-mail: shikhanema@gmail.com] \\ *Corresponding author: Amrita Ruperee
}

Received January 25, 2019; revised March 12, 2019; accepted April 7, 2019;

published September 30, 2019

\begin{abstract}
The paucity of pilot signals in Massive MIMO system is a vital issue. To accommodate substantial number of users, pilot signals are reused. This leads to interference, resulting in pilot contamination and degrades channel estimation at the Base Station (BS). Hence, mitigation of pilot contamination is exigency in Massive MIMO system. The proposed Time Shifted Pilot Signal Transmission with Pilot signal Hopping (TSPTPH), addresses the pilot contamination issue by transmitting pilot signals in non-overlapping time interval with hopping of pilot signals in each transmission slot. Hopping is carried by switching user to new a pilot signal in each transmission slot, resulting in random change of interfering users. This contributes to the change in channel coefficient, which leads to improved channel estimation at the BS and therefore enhances the efficiency of Massive MIMO system. In this system, Uplink Signal Power to Interference plus Noise Power Ratio (SINR) and data-rate are calculated for pilot signal reuse factor 1 and 3, by estimating the channel with Least Square estimation. The proposed system also reduces the uplink Signal power for data transmission of each User Equipment for normalized spectral efficiency with rising number of antennas at the BS and thus improves battery life.
\end{abstract}

Keywords: Channel estimation, Hopping, Pilot contamination, Pilot signal, Signal Power to Interference plus Noise Power Ratio. 


\section{Introduction}

In the next generation network, desire for high data-rate and reliability is increasing considerably, whilst at the same time user density and traffic intensity are increasing exponentially. Therefore, the design of the cellular network is requisite to provide a reliable and good quality of service. To a large extent, this can be taken care of by deploying a significant number of $M$ antennas at the BS, providing services to $K$ number of User Equipment (UE). Main merits of using a vast number of antennas at the BS are mentioned by [1], [2] and [3]. It not only provides reliability and quality of service but also has the following advantages:

- It reduces the radiated power of each UE to $1 / M$ for perfect Channel State information (CSI) and $1 / \sqrt{M}$ for imperfect CSI thereby, increasing the battery life.

- Channel vector is random between UE and BS, and becomes pairwise orthogonal with $M$.

- With simple signal processing, uncorrelated noise and intra-cell interference can be eliminated, and the effect of small-scale fading and thermal noise can be averaged out. Basics of MMIMO system with emphases on the challenges and opportunities are explained in [4]. In Massive MIMO (MMIMO) system channel coefficient matrix for $K$ UEs in the $I^{\text {th }}$ cell to the $n^{\text {th }}$ BS can be expressed as

$$
\mathbf{H}_{n l}=\mathbf{D}_{n l}{ }^{1 / 2} \mathbf{G}_{n l}
$$

where, $\mathbf{H}_{n l} \in \mathbb{C}^{M \times K}$ and is a combination of small-scale and large-scale fading coefficients, $\mathbf{G}$ is a $M \times K$ matrix of small-scale fading coefficients, $\mathbf{D}$ is a $K \times K$ diagonal matrix whose diagonal elements comprise the vector $[\beta]_{k}$, and $\mathbf{D}_{n l}=\operatorname{diag}\left[\beta_{n l 1}, \beta_{n l 2}, \cdots, \beta_{n l K}\right]$. The Rayleigh channel vector between $k^{\text {th }}$ user in the $l^{\text {th }}$ cell to the $i^{\text {th }}$ antenna of the $n^{\text {th }}$ BS is represented by $h_{n l k i}$ and is given as

$$
h_{n l k i}=\sqrt{\beta_{n l k}} \mathbf{g}_{n l k i}
$$

here, $\mathbf{g}_{n l k i}$ is a $1 \times M$ small-scale fading vector with $\sim C N\left(0, \mathbf{I}_{M}\right)$ unknown random variables. $\beta_{n l k}$ represents large-scale fading coefficient (geometric and shadow fading), and it is a function of $z_{n l k}$ and radial distance $r_{n l k}$ and given as

$$
\beta_{n l k}=\frac{Z_{n l k}}{r_{n l k}{ }^{\gamma}}
$$

Cells of same size may have different environments due to shadowing. Therefore, $z_{n l k}$ is a random variable having lognormal distribution about mean and changes slowly over space [5]. The quantity $10 \log _{10}\left(z_{n l k}\right)$ is Gaussian with zero mean and standard deviation of $\sigma_{\text {shad }}$. The value of $\sigma_{\text {shad }}$ varies from $6 d B$ to $12 d B$ or even higher in dense environment and $r_{n l k}$ is the radial distance between $k^{\text {th }}$ UE in the $l^{\text {th }}$ cell and the BS in the $n^{\text {th }}$ cell, $\gamma$ is the propagation path loss exponent. Therefore statistically, parameters which define the path loss due to 
large-scale fading for dynamically located UE are radial distances between interfering UEs and BS, standard deviation $\sigma$ and path loss exponent $\gamma$.

\section{Related Work}

Pilot contamination is alarming if same set of the pilot sequences is reused in multi-cell MMIMO system. Vidit et al. [6] have suggested a scheme which reduces the pilot contamination and improves the downlink SINR and sum-rate by reusing pilot signals with power control. A smart pilot assignment scheme has been proposed by Zhu et al. [7], in this scheme pilot sequence is allocated to each user after analysing the channel quality (large-scale fading coefficient) and quantifying the inter-cell interference. Researchers [8], [9] and [10] have used linear precoding techniques such as Matched Filtering (MF), Zero Forcing (ZF) and Minimum Mean Square Error (MMSE) to improve system performance such as uplink and downlink SIR and spectral efficiency in the presence of pilot contamination. Improvement in downlink performance of MMIMO system using fractional frequency reuse with MF and ZF precoding is demonstrated in our previous work [11]. Improvement in achievable data-rate when the number of antennas approaches to infinity with irregular antenna array installation is shown in [12]. Pilot contamination is severe in multi-cell MMIMO system, where users transmit pilot sequence synchronously. Kumar et al. [13] have proposed a model in which pilot are transmitted asynchronously. It reduces inter-cell interference and improves channel estimation. Time-shifted pilot sequence transmission with covariance based channel estimation scheme has been proposed by Yao et al. [14] to improve the downlink performance. To improve the performance of MMIMO system, the concept of asynchronous pilot transmission is further carried by other researchers [15], [16], [17] and [18] by using MMSE channel estimation and linear pre-coding techniques such as Matched Filtering and Zero Forcing. Jesper et al. [19] introduced a novel scheme in which users transmit pilot sequence synchronously and each user hops to new pilot sequence in each transmission slot. To improve the performance of MMIMO system in the presence of pilot contamination, a scheme is proposed in our previous work [20], where pilot signals are transmitted asynchronously, and pilot signal hopping is performed in each transmission slot. The change in interfering users in each transmission slot due to hopping, causes change in channel coefficient which results in better channel estimation. In this proposed work, we now go ahead to improve the uplink performance of MMIMO system.

In the proposed TSPTPH system, pilot signal is transmitted by UEs asynchronously with pilot hopping at each transmission slot. Due to hopping, the group of UEs contaminating the channel will change in each transmission slot and therefore improves channel estimation. In this system, pilot signal transmission is overlapped with the downlink data and channel is estimated using Least Square estimation. The results are analysed and compared with Time-Shifted Pilot signal Transmission (TSPT) [16] system and shows that there is significant improvement in uplink performance.

Notation: Matrices are represented by upper case boldface, vectors are represented by lower case boldface, $(\cdot)^{\mathrm{T}}$ is matrix transpose, $(\cdot)^{H}$ is Hermitian transpose and $x \sim C N(a ; b)$ indicates variable $x$ has a circularly symmetric complex Gaussian distribution with mean $a$ and variance $b$. 


\section{Proposed TSPTPH system model}

A multi-cell network with $L$ hexagonal cells is considered where, each cell is provided with centrally located BS having vast number of $M$ omni-directional antennas. Each cell is having $K$ users, each with single antenna and are distributed randomly in a cell. For pilot signal reuse factor $1 / N$ where, $N=1$ and 3 , there are six interfering cells. Fig. 1 Shows seven cells structure for $N=1$, and the intended UE is in the centre cell $A$ and there are six interfering cells. For $N=3$, each cluster will have 3 cells with similar configuration. In the proposed system, the pilot signal transmission is overlapped with the downlink data and simultaneously pilot signal hopping is performed in each transmission slot.

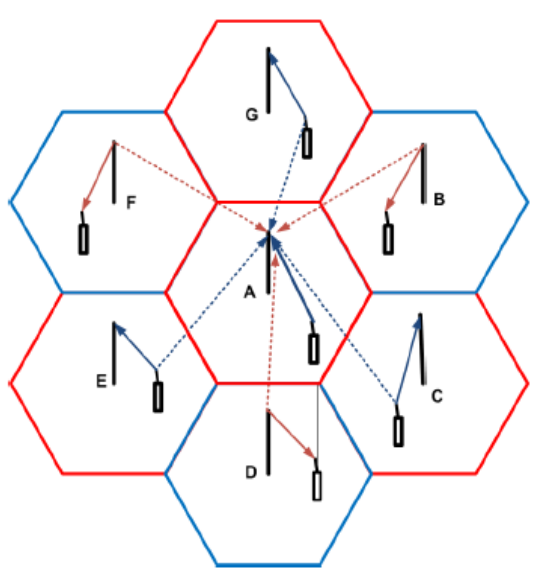

(a) Interfering users (T1)

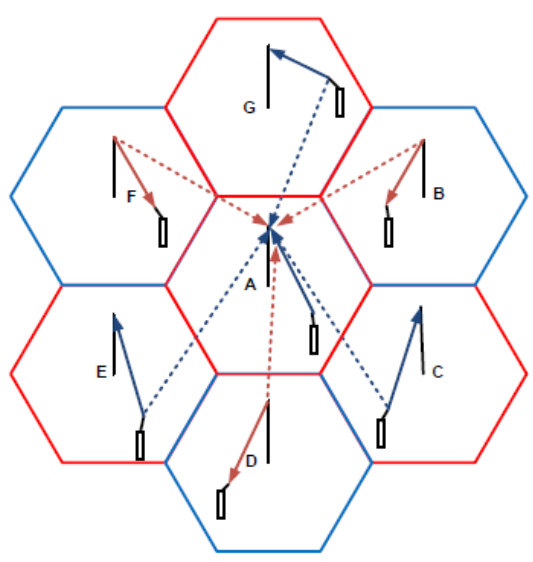

(b) Interfering users (T2)

Fig. 1. Group of users contaminating the channel

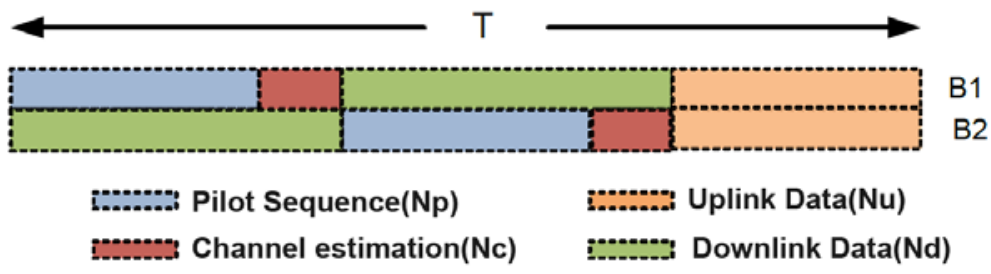

Fig. 2. Time shifted pilot sequence transmission

$B_{1}$, where UEs transmit pilot signals to their respective BS at the beginning of every coherence interval. Coherence interval of $B_{2}$ group consists of cells $B, D, F$ and starts with downlink data transmission as shown in Fig. 2. An orthogonal time-frequency pilot signal assigned to each UE within a cell. Pilot signals used by $K$ UEs can be represented by $\tau \times K$ matrix and $\psi=\left(\psi_{1}, \psi_{2}, \cdots, \psi_{K}\right)$ where, $(\tau \geq \mathrm{K})$ and $k^{\text {th }} \mathrm{UE}$ of all the cells transmits the same pilot signal $\psi_{K}$. Pilot signals are normalized such that $\psi^{H}{ }_{A k} \psi_{A k}=K \delta_{k, k}$. TDD protocol is used to get the CSI and channel vector is considered to be flat during transmission slot of $\mathrm{T}$ symbols period. In each transmission slot, four operations are performed: 
- Training phase: Each UE sends orthogonal pilot signal to the respective BS for $N_{p}$ symbol period

- Channel estimation: On receiving pilot signal, BS estimates channel vector for the corresponding user in $N_{c}$ symbol period

- Uplink data transmission: Users send uplink data to its BS for $N_{u}$ symbol period

- Downlink data transmission: BS transmits downlink data to the corresponding users for $N_{d}$ symbol period

$$
T=N_{p}+N_{c}+N_{u}+N_{d}
$$

An unique pilot signal hopping pattern which is known to BS is assigned to each UE within a cell as shown in Table 1. UEs switch to a new pilot signal in each transmission slot, this causes random change in group of interfering users. Therefore, the channel coefficient i.e. the large-scale fading coefficient $\beta$ which is a function of $z$ (log-normal distributed random variable) and $r$ (radial distance between UE and BS) will also change in every transmission slot as depicted in Fig. 1. Thus, in each transmission slot, channel is contaminated by dynamically changing interfering users due to pilot hopping. This randomization of the interfering users significantly reduces the channel estimation error. Whereas, in conventional MMIMO system and TSPT system channel coefficients between UEs and BS will remain same since there is no change in the interfering users.

Table 1. Pilot sequence hopping pattern

\begin{tabular}{|c|c|c|c|c|c|c|}
\hline Users & $\mathrm{T}_{1}$ & $\mathrm{~T}_{2}$ & $\mathrm{~T}_{3}$ & $\mathrm{~T}_{4}$ & $\mathrm{~T}_{5}$ & $\mathrm{~T}_{6}$ \\
\hline 01 & $\Psi_{1}$ & $\Psi_{5}$ & $\Psi_{4}$ & $\Psi_{3}$ & $\Psi_{2}$ & $\Psi_{6}$ \\
\hline 02 & $\Psi_{3}$ & $\Psi_{2}$ & $\Psi_{6}$ & $\Psi_{5}$ & $\Psi_{4}$ & $\Psi_{1}$ \\
\hline 03 & $\Psi_{4}$ & $\Psi_{6}$ & $\Psi_{5}$ & $\Psi_{2}$ & $\Psi_{1}$ & $\Psi_{3}$ \\
\hline 04 & $\Psi_{5}$ & $\Psi_{4}$ & $\Psi_{3}$ & $\Psi_{6}$ & $\Psi_{5}$ & $\Psi_{2}$ \\
\hline 04 & $\Psi_{2}$ & $\Psi_{3}$ & $\Psi_{1}$ & $\Psi_{1}$ & $\Psi_{6}$ & $\Psi_{4}$ \\
\hline 05 & $\Psi_{6}$ & $\Psi_{1}$ & $\Psi_{2}$ & $\Psi_{4}$ & $\Psi_{3}$ & $\Psi_{5}$ \\
\hline
\end{tabular}

\subsection{Training Phase}

In training phase, UEs of group $B_{1}$ cells transmit the pilot signal, and BSs of group $B_{2}$ cells transmit downlink data at the beginning of each coherence interval. The pilot signal received at the $\mathrm{BS} A$ is $\mathbf{Y}_{A}$ given as

$$
\mathbf{Y}_{A}=\sum_{i \in B_{1}} \sum_{k=1}^{K} \sqrt{\rho_{p}} \sqrt{\beta_{A i k}} \boldsymbol{\psi}_{k} \mathbf{g}_{A i k}+\sum_{m \in B_{2}} \sum_{k=1}^{K} \sqrt{\rho_{d}} \sqrt{\lambda_{A m}} \mathbf{q}_{m k} \mathbf{w}_{m k} \mathbf{J}_{A m}+\mathbf{V}_{A}
$$

Where, $\mathbf{Y}_{A}$ is $K \times M$ received signal matrix at the BS $A, \beta_{\text {Aik }}$ and $\mathbf{g}_{\text {Aik }}$ are large-scale and small-scale fading coefficient between $k^{\text {th }}$ user of $i^{\text {th }}$ cell and $A^{\text {th }} \mathrm{BS}, \lambda_{A m}$ and $\mathbf{J}_{A m}$ are large-scale and small-scale fading coefficient between the BS $A$ and the BS $m$, and $\mathbf{J}_{A m} \sim C N\left(0, \mathbf{I}_{M \times M}\right)$. Since, BSs are stationary, the channel characteristic between BSs 
change very slowly. $\boldsymbol{\psi}_{k}$ is $K \times 1$ pilot signal vector of $k^{\text {th }}$ UE, $\rho_{p}$ is the measure of pilot signal power to noise variance ratio and $\rho_{d}$ is the ratio of downlink data power to noise variance. Here, noise variance is considered to be one, $\mathbf{q}_{m k}$ is $K \times 1$ message bearing symbol for the $k^{\text {th }}$ UE from the $m^{\text {th }}$ BS and $\mathbf{q}_{m k} \sim C N\left(0, \mathbf{I}_{K}\right)$. It means, symbol vectors are uncorrelated and having variance one i.e. $E\left\{\mathbf{q}_{m k} \mathbf{q}_{m k}{ }^{H}\right\}=\mathbf{I}_{K}, \mathbf{V}_{A}$ is $K \times M$ additive noise matrix, which is defined as IID, $C N\left(0, \mathbf{I}_{K M}\right)$ and $\mathbf{w}_{m k}$ is $M \times 1$ pre-coding vectors for the $k^{\text {th }}$ user from the $m^{\text {th }}$ BS.

\subsection{Channel Estimation}

On receiving the pilot signals, the $A^{\text {th }}$ BS estimates the channel vector for its $k^{\text {th }}$ UE using the Least Square estimation by correlating it with known pilot signal and is given by

$$
\begin{gathered}
\hat{\mathbf{g}}_{A A k^{\prime}}=\boldsymbol{\psi}_{k^{\prime}}{ }^{H} \times \mathbf{Y}_{A} \\
\hat{\mathbf{g}}_{A A k^{\prime}}=\sqrt{\rho_{p}} \sum_{i \in B_{1}} \sum_{k=1}^{K} \sqrt{\beta_{A i k}} \mathbf{g}_{A i k}, K+\sum_{m \in B_{2}} \sum_{k=1}^{K} \sqrt{\rho_{d} \lambda_{A m k^{\prime}}} \boldsymbol{\psi}_{k^{\prime}}{ }^{H} \mathbf{J}_{A m k^{\prime}} \mathbf{w}_{m k^{\prime}} \mathbf{q}_{m k^{\prime}}+\boldsymbol{\psi}_{k^{\prime}}{ }^{H} \mathbf{V}_{A}
\end{gathered}
$$

The Estimated channel for $k^{\text {th }}$ UE is given in equation. 7, it shows that channel is contaminated by the pilot signals transmitted by UEs of group $B_{1}$ cells (first term), downlink data transmitted by BSs belonging to group $B_{2}$ (second term) and the noise. The first term $\beta_{\text {Aik' }}$ is the function of radial distance between UE and BS and will vary in each transmission slot as the group of UEs changes randomly due to pilot signal hopping. The covariance of the estimated channel is calculated by considering that, every random variable in equation. 7 is uncorrelated and therefore covariance is given as

$$
\begin{aligned}
& \mathbf{C}_{\hat{\mathbf{g}}_{A A k^{\prime}} \hat{g}_{A A k^{\prime}}}=\sum_{i \in B_{1}} \rho_{p} \beta_{A i k^{\prime}} K^{2} \mathbf{I}_{M}+\sum_{m \in B_{2}} \sum_{k=1}^{K} \rho_{d} \lambda_{A m k^{\prime}} K \mathbf{I}_{M}+K \mathbf{I}_{M} \\
& \mathbf{C}_{\mathrm{g}_{A A k} \cdot \mathbf{g}_{A A k^{\prime}}}=\phi_{A A k}, K \mathbf{I}_{M}
\end{aligned}
$$

where, $\quad \phi_{A A k^{\prime}}=\sum_{i \in B_{1}} \rho_{p} \beta_{A i k^{\prime}} K+\sum_{m \in B_{2}} \sum_{k=1}^{K} \rho_{d} \lambda_{A m k^{\prime}}+1$

The estimation error vector $\overline{\mathbf{g}}_{A A k^{\prime}}=\hat{\mathbf{g}}_{A A k^{\prime}}-\mathbf{g}_{A A k^{\prime}}$, here $\overline{\mathbf{g}}_{A A k^{\prime}}$ and $\hat{\mathbf{g}}_{A A k^{\prime}}$ are uncorrelated therefore the covariance matrix of $C_{\overline{\mathbf{g}}_{A A k} \cdot \overline{\mathbf{g}}_{A A k^{\prime}}}$ is given as $\left(1-\phi_{A A k^{\prime}} K\right) \mathbf{I}_{M}$. It is considered that estimated channel is having Gaussian distribution, thus $\hat{\mathbf{g}}_{A A k^{\prime}}$ and $\overline{\mathbf{g}}_{A A k^{\prime}}$ have distribution $C N\left(0, \phi_{A A k^{\prime}} K \mathbf{I}_{M}\right)$ and $C N\left(0,\left(1-\phi_{A A k^{\prime}} K \mathbf{I}_{M}\right)\right)$ respectively.

\subsection{Uplink data transmission}

All the UEs of group $B_{1}$ and $B_{2}$ cells transmit uplink data simultaneously to their respective BSs. And BSs receive $M \times 1$ vectors of message symbol from each UE.

Therefore $A^{\text {th }}$ BS receives the uplink data transmitted by its $k^{\text {th }}$ UE is given as 


$$
d_{A k^{\prime}}=\sum_{i \in B_{1}} \sum_{k=1}^{K} \sqrt{\rho_{u}} \sqrt{\beta_{A i k}} \mathbf{g}_{A i k^{\prime}} d_{i k}+\sum_{m \in B_{2}} \sum_{k=1}^{K} \sqrt{\rho_{u}} \sqrt{\beta_{A m k^{\prime}}} \mathbf{g}_{A m k^{\prime}}, d_{m k}+\mathbf{n}_{A}
$$

Here, $\mathbf{n}_{A}$ is $1 \times M$ additive noise vector between the BS $A$ and the $k^{\text {th }}$ user with entries IID, $C N\left(0, \mathbf{I}_{M}\right), \rho_{u}$ is the ratio of uplink data transmission power to noise variance and $d_{i k}$ is the uplink data transmitted from $k^{\text {th }}$ UE. Maximum Ratio Combining (MRC) is used at the BS to calculate the estimated data which is a function of $\hat{\mathbf{g}}_{\text {AAk' }}$. Therefore, estimated data for the $k^{\text {th }}$ user of cell $A$ is given as

$$
d_{A A k^{\prime}}=d_{A k^{\prime}} \mathbf{f}_{A k^{\prime}}
$$

where, $\mathbf{f}_{A k^{\prime}}$ is the beam forming vector and it is defined as

$$
\mathbf{f}_{A k^{\prime}}=\frac{\hat{\mathbf{g}}_{A A k^{\prime}}^{H}}{\sqrt{K}\left\|\hat{\mathbf{g}}_{A A k^{\prime}}^{H}\right\|}
$$

From equations. 11 and 12 the estimated data for $k^{\text {th }}$ user is obtained as

$$
d_{A A k^{\prime}}=\sum_{i \in B_{1}} \sum_{k=1}^{K} \sqrt{\rho_{u}} \sqrt{\beta_{A i k^{\prime}}} \mathbf{g}_{A i k^{\prime}} d_{i k} \mathbf{f}_{A k^{\prime}}+\sum_{m \in B_{2}} \sum_{k=1}^{K} \sqrt{\rho_{u}} \sqrt{\beta_{A m k^{\prime}}} \mathbf{g}_{A m k^{\prime}} d_{m k} \mathbf{f}_{A k^{\prime}}+\mathbf{n}_{A} \mathbf{f}_{A k^{\prime}}
$$

SINR for the $k^{\text {th }}$ UE of cell $A$ is defined as $\frac{S_{a}}{N_{a}}$. Signal power $S_{a}$ is illustrated as

$$
S_{a}=\rho_{u} \beta_{A A k^{\prime}}\left|E\left[\mathbf{g}_{A A k^{\prime}} \mathbf{f}_{A k^{\prime}}\right]\right|^{2}
$$

The achievable uplink data rate for the $k^{\text {th }}$ UE of cell $A$ is given as

$$
R_{A k^{\prime}}^{U p}=\frac{N_{u}}{T} E\left[\log _{2}\left(1+\operatorname{SINR}_{\mathrm{AAk} k^{\prime}}^{U p}\right)\right]
$$

where, $N_{u}$ is the uplink data symbol period. The Complexity of expectation in equation. 16 is reduced by using Jensen's inequality [16] to calculate estimated achievable uplink data-rate and is given as

$$
\begin{gathered}
R_{A k^{\prime}}^{U p} \geq \bar{R}_{A k^{\prime}}^{U p} \\
\bar{R}_{A k^{\prime}}^{U p}=\frac{N_{u}}{T} \log _{2}\left(\left(E\left[\frac{N_{a}}{S_{a}}\right]\right)^{-1}\right)
\end{gathered}
$$

Therefore, $S_{a}$ can be expressed as

$$
S_{a}=\rho_{u} K \beta_{A A k^{\prime}} \phi_{A A k^{\prime}}(M-1)
$$

And the expected value of $\frac{N_{a}}{S_{a}}$ can be expanded as

$$
E\left[\frac{N_{a}}{S_{a}}\right]=\left[\begin{array}{l}
\rho_{u} \beta_{A A k^{\prime}} E\left[\left|\overline{\mathbf{g}}_{A A k^{\prime}} \mathbf{f}_{A k^{\prime}}\right|^{2} / S_{a}\right]+\sum_{i \neq A, i \in L} \rho_{u} \beta_{A i k^{\prime}} E\left[\left|\mathbf{g}_{A i k^{\prime}} \mathbf{f}_{A k^{\prime}}\right|^{2} / S_{a}\right] \\
+\sum_{i \in L} \sum_{k \neq k^{\prime}}^{K} \rho_{u} \beta_{A i k^{\prime}} E\left[\left|\mathbf{g}_{A i k^{\prime}} \mathbf{f}_{A k^{\prime}}\right|^{2} / S_{a}\right]+E\left[\left|n_{A} \mathbf{f}_{A k^{\prime}}\right|^{2} / S_{a}\right]
\end{array}\right]
$$


By using equations. 19 and 20, the closed form expression for Uplink SINR is inferred as

$$
\operatorname{SINR}_{A A k^{\prime}}^{U p}=\frac{\rho_{u} K \beta_{A A k^{\prime}} \phi_{A A k^{\prime}}(M-1)}{\left[\begin{array}{l}
\left.\rho_{u} \beta_{A A k^{\prime}}\left[\left(1-\phi_{A A k^{\prime}} K\right)\right]+\sum_{i \neq A, i \in L} \rho_{u} \beta_{A i k^{\prime}}\left[\left(\phi_{i k^{\prime}} K(M-1)+1\right)\right]\right] \\
+\sum_{i \in L} \sum_{k \neq k^{\prime}}^{K} \rho_{u} \beta_{A i k^{\prime}}+1
\end{array}\right]}
$$

The expression for achievable uplink data-rate for the $k^{\text {th }} \mathrm{UE}$ of cell $A$ is given as

$$
\bar{R}_{A A k^{\prime}}^{U p}=\frac{N_{u}}{T} \log _{2}\left(1+\frac{\rho_{u} K \beta_{A A k^{\prime}} \phi_{A A k^{\prime}}(M-1)}{\left[\begin{array}{l}
\left.\rho_{u} \beta_{A A k^{\prime}}\left[\left(1-\phi_{A A k^{\prime}} K\right)\right]+\sum_{i \in L} \sum_{k \neq k^{\prime}}^{K} \rho_{u} \beta_{A i k^{\prime}}+1\right] \\
+\sum_{i \neq A, i \in L} \rho_{u} \beta_{A i k^{\prime}}\left[\left(\phi_{i k^{\prime}} K(M-1)+1\right)\right]
\end{array}\right]}\right)
$$

In TSPT, interfering users remain same as there is no hopping whereas in TSPTPH, due pilot signal hopping, interfering users change in each transmission slot. From equation. 21 and 22, it is observed that SINR and data-rate are function of $\beta_{\text {Aik }}, K$ and $M$, first term of the denominator is the channel estimation error and second term is the interference caused by UEs of group $B_{1}$ and $B_{2}$ cells transmitting the same pilot signal except cell $A$. And, these interfering UEs change dynamically in each transmission slot due to the pilot signal hopping. Therefore, $\beta_{\text {Aik' }}$ and $\phi_{i k^{\prime}}$ are function of radial distance between UEs and BS also change as explained (see Appendix). Third term is the interference caused by users of group $B_{1}$ and $B_{2}$, where $k \neq k$ ' which also varies in each transmission slot due to pilot hopping and fourth term is the noise variance.

\section{Simulation Results and analysis}

The proposed TSPTPH system is evaluated for $K=8$ and $K=15$, pilot signal hopping is done for 30 and 40 transmission slots and results are compared with TSPT for reuse $N=1$ and $N=3$, by varying the BS antennas from 10 to 250 . Simulations are carried out in MATLAB for 1500 trials and average values are finally used for analysis of the results. Cells are considered to be of the same size with radius of $1.5 \mathrm{~km}$ and having no users within $100 \mathrm{~m}$ radius of cell. Each cell is provided with equal number of UEs that are distributed randomly. $\rho_{p}=10 \mathrm{~dB}, \rho_{u}=10 \mathrm{~dB}$ and $\rho_{d}=20 \mathrm{~dB}$ are the measures of SNR during pilot signal, uplink and downlink data transmission phase respectively. The noise variance is considered to be one and all the transmission slots have $T=17, N_{u}=6, N_{d}=6, N_{c}=2, N_{p}=3$. 
In mobile system, propagation environment of cells may have different characteristics though they are of the same size. Therefore, it is required to calculate variation about mean. In [21], path loss $L_{p}(d)$ is a random variable having log-normal distribution about the mean, which is distant-dependent value $\overline{L_{p}}(d)$. Thus $L_{p}(d)$ can be expressed as

$$
L_{p}(d)(d B)=L_{s}\left(d_{0}\right)(d B)+10 \gamma \log \left(r / d_{0}\right)+X_{\sigma}(d B)
$$

here, $X_{\sigma}$ is zero-mean Gaussian random variable with standard deviation $\sigma_{\text {shad }}(d B) . X_{\sigma}$ is equivalent to $Z$ and dependent on cell environment and cell size. Thus, analysis of the Rayleigh fading channel is carried out for three path loss models taking into consideration shadow fading $\sigma_{\text {shad }}(d B)=0 d B, 8 d B$ and $12 d B$ with path-loss exponent $\gamma=3.8$.

The pseudo code of the implementation is given below Algorithm 1. Uplink SINR and data-rate for the three path loss models for $K=8$ with 40 hops and 30 hops for reuse factor $N=1$ and $N=3$, are shown in Fig. 3 and Fig. 4. The corresponding observations are inferred in Table 2 - Table 5. The analysis of the observations is given below.

Reuse factor $N=1$

- Performance of TSPTPH is slightly better for $M<110$ both for 30 hops and 40hops for $\sigma_{\text {shad }}=0 d B$. It starts decreasing slowly for 30 hops when $M>200$ for both TSPTPH and TSPT. For $\sigma_{\text {shad }}=8 d B$, both systems perform nearly same. As the effect of fading increases i.e. $\sigma_{\text {shad }}=12 d B$, TSPTPH performs much better and shows average improvement of $4.2 \%$ for 40 hops. For 30 hops, TSPT performs better for $M>150$.

Reuse factor $N=3$

- TSPTPH performs significantly better till $M<220$ for $\sigma_{\text {shad }}=0 d B$ with 40 hops and shows $3.4 \%$ improvement and it is $1.8 \%$ for 30 hops when $M>90$. For $\sigma_{\text {shad }}=8 d B$, performance of TSPTPH much better than TSPT and increases continuously with $\mathrm{M}$ and shows 3\% improvement for 40 hops, whereas for 30 hops TSPTPH performs well till $M<100$ and then TSPT performs good and shows improvement of $2.5 \%$ with respect to TSPTPH. For $\sigma_{\text {shad }}=12 d B$, TSPTPH performs much better and shows improvement of $4 \%$ over TSPT for 40 hops whereas for 30 hops, performance of TSPT is better for $M<130$ and then after TSPTPH shows improvement of $1.5 \%$.

Table 2. Uplink SINR (dB) K=8, N=1

\begin{tabular}{|c|c|c|c|c|c|c|c|c|}
\hline \multirow{2}{*}{$\begin{array}{c}\text { Path-loss } \\
\text { model } \\
\sigma_{\text {shad }}\end{array}$} & \multicolumn{3}{|c|}{ Number of hops $=40$} & \multicolumn{3}{c|}{ Number of hops $=30$} \\
\cline { 2 - 9 } & \multicolumn{2}{|c|}{$\mathrm{M}=100$} & \multicolumn{2}{c|}{$\mathrm{M}=200$} & \multicolumn{2}{c|}{$\mathrm{M}=100$} & \multicolumn{2}{c|}{$\mathrm{M}=200$} \\
\hline $0 \mathrm{dBPT}$ & 39.1 & $39 P T \mathrm{~B}$ & $\mathrm{TSPT}$ & $\mathrm{TSPTPH}$ & $\mathrm{TSPT}$ & $\mathrm{TSPTPH}$ & $\mathrm{TSPT}$ & $\mathrm{TSPTPH}$ \\
\hline $8 \mathrm{~dB}$ & 40.25 & 39.9 & 43 & 41.8 & 39.3 & 40.7 & 43.9 & 43.3 \\
\hline $12 \mathrm{~dB}$ & 38.8 & 41.8 & 43.1 & 43.5 & 42.1 & 41.6 & 44.9 & 43.8 \\
\hline
\end{tabular}


Table 3. Uplink data-rate (bps / hz) K=8, N=1

\begin{tabular}{|c|c|c|c|c|c|c|c|c|}
\hline \multirow{2}{*}{$\begin{array}{c}\text { Path-loss } \\
\text { model } \\
\sigma_{\text {shad }}\end{array}$} & \multicolumn{3}{|c|}{ Number of hops $=40$} & \multicolumn{4}{c|}{ Number of hops = 30 } \\
\cline { 2 - 9 } & \multicolumn{2}{|c|}{$\mathrm{M}=100$} & \multicolumn{2}{|c|}{$\mathrm{M}=200$} & \multicolumn{2}{c|}{$\mathrm{M}=100$} & \multicolumn{2}{c|}{$\mathrm{M}=200$} \\
\hline $0 \mathrm{~dB}$ & 4.58 & 4.62 & 5.03 & 4.9 & 4.67 & 4.78 & 5.15 & 5.07 \\
\hline $8 \mathrm{~dB}$ & 4.72 & 4.68 & 5.15 & 5.1 & 4.94 & 4.87 & 5.26 & 5.14 \\
\hline $12 \mathrm{~dB}$ & 4.56 & 4.78 & 5.08 & 5.28 & 5.13 & 4.7 & 5.3 & 5.1 \\
\hline
\end{tabular}

Table 4. Uplink SINR (dB) $\mathrm{K}=8, \mathrm{~N}=3$

\begin{tabular}{|c|c|c|c|c|c|c|c|c|}
\hline \multirow{2}{*}{$\begin{array}{c}\text { Path-loss } \\
\text { model } \\
\sigma_{\text {shad }}\end{array}$} & \multicolumn{3}{|c|}{ Number of hops $=40$} & \multicolumn{4}{c|}{ Number of hops = 30 } \\
\cline { 2 - 9 } & \multicolumn{2}{|c|}{$\mathrm{M}=100$} & \multicolumn{2}{|c|}{$\mathrm{M}=200$} & \multicolumn{2}{c|}{$\mathrm{M}=100$} & \multicolumn{2}{c|}{$\mathrm{M}=200$} \\
\hline $0 \mathrm{dBPT}$ & 44.4 & TSPTPH & TSPT & TSPTPH & TSPT & TSPTPH & TSPT & TSPTPH \\
\hline $8 \mathrm{~dB}$ & 44.4 & 45.3 & 51.3 & 52.4 & 45.65 & 45.87 & 50 & 53 \\
\hline $12 \mathrm{~dB}$ & 42 & 45.3 & 50.4 & 51.5 & 44.75 & 44.63 & 52 & 49.5 \\
\hline
\end{tabular}

Table 5. Uplink data-rate (bps / hz) $\mathrm{K}=8, \mathrm{~N}=3$

\begin{tabular}{|c|c|c|c|c|c|c|c|c|}
\hline \multirow{2}{*}{$\begin{array}{c}\text { Path-loss } \\
\text { model } \\
\sigma_{\text {shad }}\end{array}$} & \multicolumn{3}{|c|}{ Number of hops $=40$} & \multicolumn{3}{c|}{ Number of hops = 30 } \\
\cline { 2 - 9 } & TSPT & TSPTPH & TSPT & TSPTPH & TSPT & TSPTPH & TSPT & TSPTPH \\
\hline $0 \mathrm{~dB}$ & 5.2 & 5.43 & 6.01 & 6.14 & 5.35 & 5.38 & 5.9 & 6.24 \\
\hline $8 \mathrm{~dB}$ & 5.2 & 5.3 & 5.8 & 6.05 & 5.248 & 5.234 & 6.08 & 5.8 \\
\hline $12 \mathrm{~dB}$ & 4.95 & 5.3 & 5.95 & 6.03 & 5.15 & 5.0 & 5.8 & 6.1 \\
\hline
\end{tabular}

Algorithm 1. Psudeo Code of the implementation

Assign pilot sequence as shown in Table I

Select number of hops

Select number of iterations

for reuse factor $\mathrm{N}=1$ and 3

for iteration $=1$ to number of iterations for transmission slots $=1$ to number of hops for antennas $=1$ to 250

Calculate channel estimation

Calculate Beamforming vector

Calculate Uplink SINR

Calculate Uplink achievable data-rate

Calculate Uplink transmission power (SNR)

end

end

end

end

Average uplink SINR for iterations

Average uplink data-rate for iterations

Average uplink transmission power for iterations

Similarly Uplink SINR and data-rate for the three path- loss models for reuse factor $N=1$ 
and $N=3$, for $K=15$ with 40 hops and 30 hops are shown in Fig. 5 and Fig. 6. The corresponding observations are depicted in Table 6 - 9 respectively. Observations are

Reuse factor $N=1$

- Performance of TSPT is better for $M>190$ and then TSPTPH start improving for 40 hops with $\sigma_{\text {shad }}=0 d B$. For $\sigma_{\text {shad }}=8 d B$, TSPTPH performs significantly better and shows $5 \%$ improvement for 40 hops and $4 \%$ for 30 hops over TSPT. In severe fading environment i.e. $\sigma_{\text {shad }}=12 d B$, TSPTPH gives $3 \%$ improvement over TSPT for 40 hops and for 30 hops TSPT performance improves by $6 \%$.

Reuse factor $N=3$

- TSPTPH performs much better and improves performance by $4.5 \%$ for 40 hops with $\sigma_{\text {shad }}=0 \mathrm{~dB}$, whereas for 30hops TSPTPH shows 3\% improvement for $M<140$. For $\sigma_{\text {shad }}=8 d B$, performance of TSPTPH shows $2 \%$ for 40 hops and $3.5 \%$ for 30 hops improvement over TSPT. For $\sigma_{\text {shad }}=12 d B$, TSPTPH gives $2 \%$ improvement for 30 hops and for 40 hops it performs well for $M>110$ with 3\% improvement over TSPT.

Table 6. Uplink SINR (dB) K=15, $\mathrm{N}=1$

\begin{tabular}{|c|c|c|c|c|c|c|c|c|}
\hline \multirow{2}{*}{$\begin{array}{c}\text { Path-loss } \\
\text { model } \\
\sigma_{\text {shad }}\end{array}$} & \multicolumn{3}{|c|}{ Number of hops $=40$} & \multicolumn{3}{c|}{ Number of hops = 30 } \\
\cline { 2 - 9 } & MSPT $=100$ & TSPTPH & TSPT & TSPTPH & TSPT & TSPTPH & TSPT & TSPTPH \\
\hline $0 \mathrm{~dB}$ & 43.7 & 41.5 & 44.5 & 44.7 & 39.7 & 42 & 45.1 & 42.25 \\
\hline $8 \mathrm{~dB}$ & 41 & 42.5 & 44.5 & 47 & 41 & 42.9 & 43.8 & 45.6 \\
\hline $12 \mathrm{~dB}$ & 39.4 & 39.8 & 44 & 46.5 & 41 & 37 & 48 & 46.2 \\
\hline
\end{tabular}

Table 7. Uplink data-rate (bps / hz) $\mathrm{K}=15, \mathrm{~N}=1$

\begin{tabular}{|c|c|c|c|c|c|c|c|c|}
\hline $\begin{array}{c}\text { Path-loss } \\
\text { model } \\
\sigma_{\text {shad }}\end{array}$ & \multicolumn{3}{|c|}{ Number of hops $=40$} & \multicolumn{3}{c|}{ Number of hops = 30 } \\
\cline { 2 - 9 } & \multicolumn{2}{|c|}{$\mathrm{M}=100$} & \multicolumn{2}{c|}{$\mathrm{M}=200$} & \multicolumn{2}{c|}{$\mathrm{M}=100$} & \multicolumn{2}{|c|}{$\mathrm{M}=200$} \\
\hline $0 \mathrm{~dB}$ & 5.1 & 4.85 & 5.22 & 5.24 & 4.65 & 5.0 & 5.28 & 5.3 \\
\hline $8 \mathrm{~dB}$ & 4.8 & 5.0 & 5.2 & 5.5 & 4.8 & 5.03 & 5.13 & 5.35 \\
\hline $12 \mathrm{~dB}$ & 4.62 & 4.66 & 5.18 & 5.45 & 4.8 & 4.37 & 5.6 & 5.4 \\
\hline
\end{tabular}

Table 8. Uplink SINR (dB) $\mathrm{K}=15, \mathrm{~N}=3$

\begin{tabular}{|c|c|c|c|c|c|c|c|c|}
\hline \multirow{2}{*}{$\begin{array}{c}\text { Path-loss } \\
\text { model } \\
\sigma_{\text {shad }}\end{array}$} & \multicolumn{3}{|c|}{ Number of hops $=40$} & \multicolumn{3}{c|}{ Number of hops = 30 } \\
\cline { 2 - 9 } & \multicolumn{2}{|c|}{$\mathrm{M}=100$} & \multicolumn{2}{|c|}{$\mathrm{M}=200$} & \multicolumn{2}{c|}{$\mathrm{M}=100$} & \multicolumn{2}{c|}{$\mathrm{M}=200$} \\
\hline $0 \mathrm{~dB}$ & 46.3 & 48.6 & 52 & 54 & 47.8 & 48.8 & 54.9 & 53.8 \\
\hline $8 \mathrm{~dB}$ & 46.4 & 48 & 51.25 & 51.4 & 45.5 & 46.5 & 54.8 & 57.2 \\
\hline $12 \mathrm{~dB}$ & 46.4 & 46.2 & 54.8 & 56.3 & 42.7 & 43.8 & 50.5 & 51.7 \\
\hline
\end{tabular}

Table 9. Uplink data-rate (bps / hz) K=15, N=3

\begin{tabular}{|c|c|c|c|c|c|c|c|c|}
\hline \multirow{2}{*}{$\begin{array}{c}\text { Path-loss } \\
\sigma_{\text {shad }}\end{array}$} & \multicolumn{3}{|c|}{ Number of hops $=40$} & \multicolumn{3}{c|}{ Number of hops $=30$} \\
\cline { 2 - 9 } & \multicolumn{2}{|c|}{$\mathrm{M}=100$} & \multicolumn{2}{|c|}{$\mathrm{M}=200$} & \multicolumn{2}{c|}{$\mathrm{M}=100$} & \multicolumn{2}{c|}{$\mathrm{M}=200$} \\
\hline & TSPT & TSPTPH & TSPT & TSPTPH & TSPT & TSPTPH & TSPT & TSPTPH \\
\hline $0 \mathrm{~dB}$ & 5.43 & 5.7 & 6.1 & 6.32 & 5.62 & 5.73 & 6.43 & 6.3 \\
\hline $8 \mathrm{~dB}$ & 5.43 & 5.64 & 6.01 & 6.025 & 5.33 & 5.45 & 6.4 & 6.7 \\
\hline $12 \mathrm{~dB}$ & 5.44 & 5.42 & 6.4 & 6.6 & 5.01 & 5.14 & 5.96 & 6.13 \\
\hline
\end{tabular}




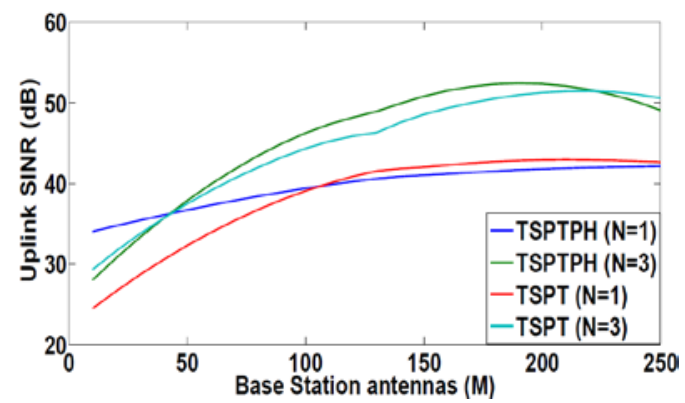

(a) $\sigma=0 \mathrm{~dB}$

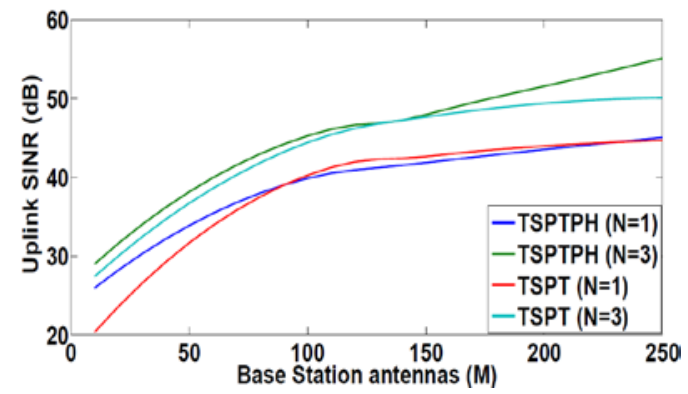

(b) $\sigma=8 d B$

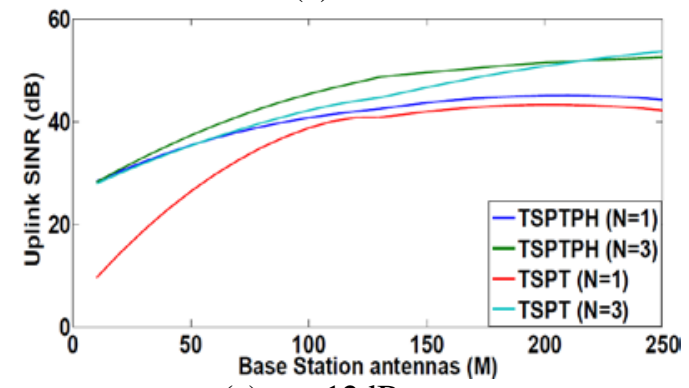

(c) $\sigma=12 \mathrm{~dB}$

Uplink SINR (40 hops)

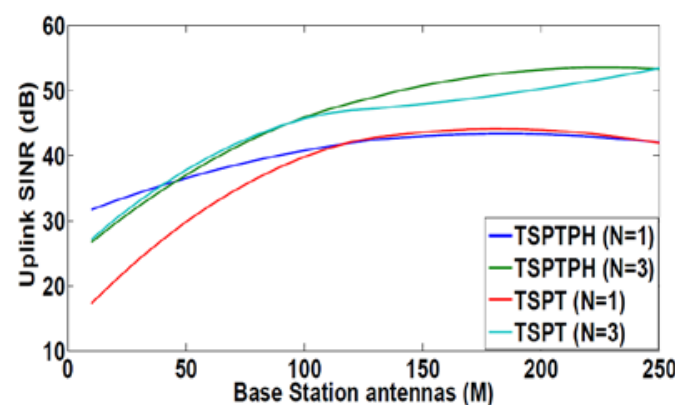

(a) $\sigma=0 \mathrm{~dB}$

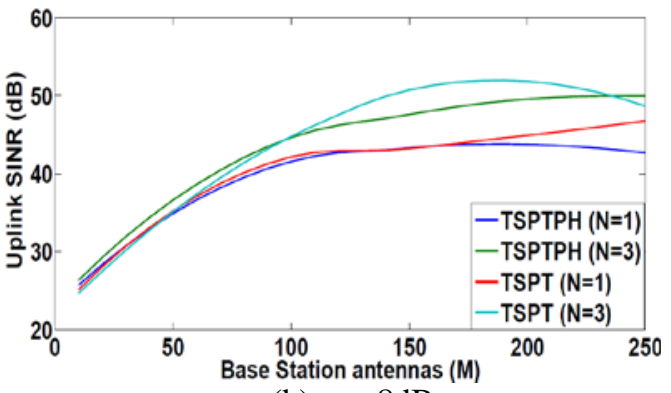

(b) $\sigma=8 \mathrm{~dB}$

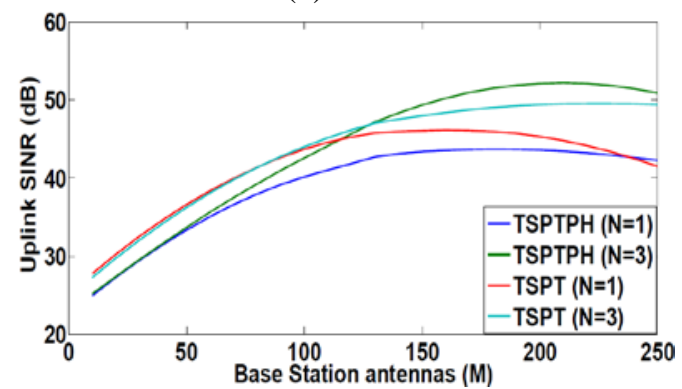

(c) $\sigma=12 \mathrm{~dB}$

Uplink SINR (30 hops)

Fig. 3. Uplink SINR with respect to antennas for $\mathrm{K}=8,40$ hops and 30 hops

From the above analysis it is observed that performance of uplink is better for $N=3$. In this case, the interfering cells are far apart i.e. $\sqrt{3 N}$ from the central cell, therefore, distance between the UE and the interfering cells UEs is very large. 


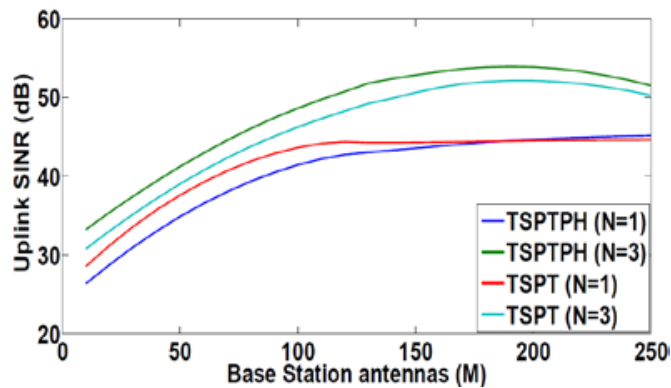

(a) $\sigma=0 \mathrm{~dB}$

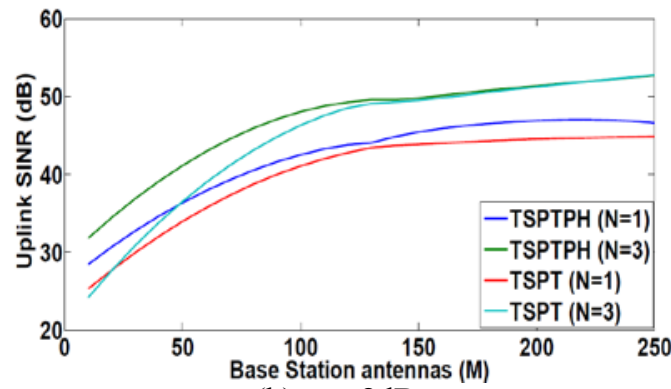

(b) $\sigma=8 \mathrm{~dB}$

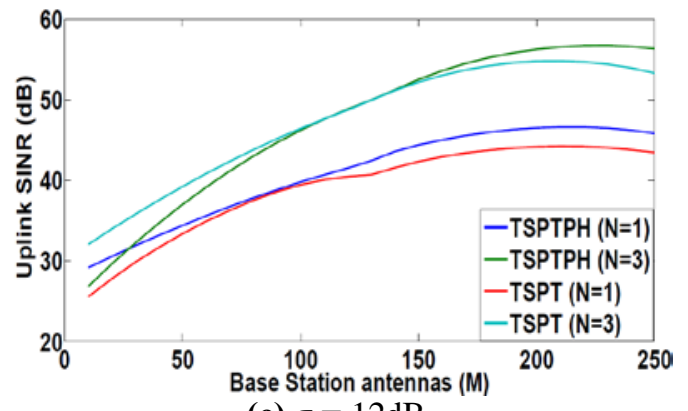

(c) $\sigma=12 \mathrm{~dB}$

Uplink SINR (40 hops)

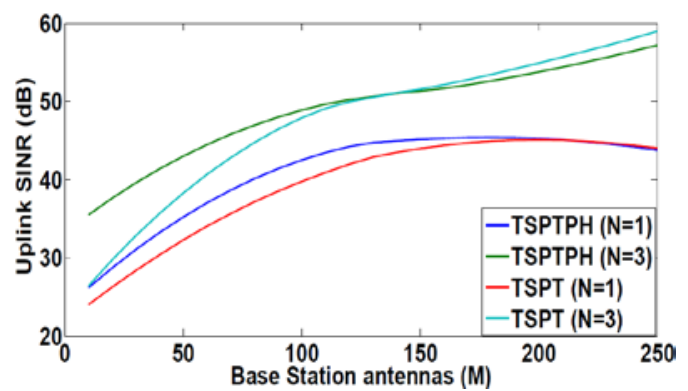

(a) $\sigma=0 \mathrm{~dB}$

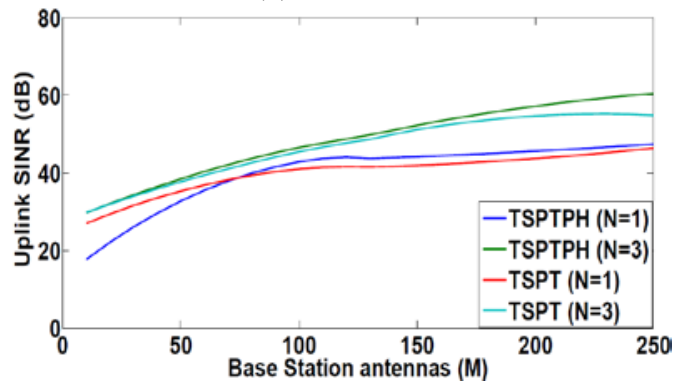

(b) $\sigma=8 \mathrm{~dB}$

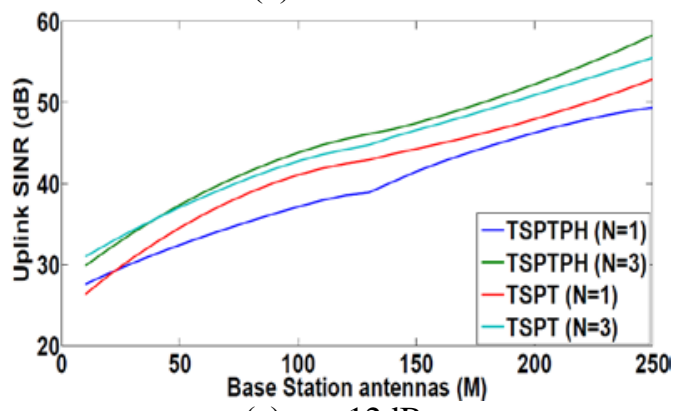

(c) $\sigma=12 \mathrm{~dB}$

Uplink SINR (30 hops)

Fig. 5. Uplink SINR with respect to BS antennas for $\mathrm{K}=15$, 40 hops and 30 hops

Thus, it reduces the denominator term $\beta_{\text {Aik' }}$ of equations. 10, 21 and 22 which results in increase in SINR and thus data-rate. Further, the analysis of uplink data transmission power required per UE for imperfect CSI to achieve spectral efficiency of $1 \mathrm{bit} / \mathrm{sec} / \mathrm{hz}$ with respect to BS antennas for $K=15$, is depicted in Fig. 7. It shows that, as the number of BS antenna increases, uplink SNR decreases. Uplink SNR nearly same for both the schemes for $N=3$ with 40 hops and 30 hops whereas, for $N=1$, TSPTPH performs better with 40 hops. It is also noticed that, as the effect of shadowing increases, UE has to raise their SNR for uplink data transmission to achieve the same spectral efficiency. 


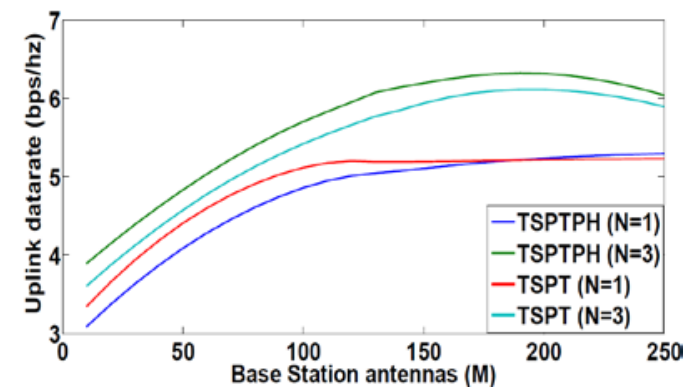

(a) $\sigma=0 \mathrm{~dB}$

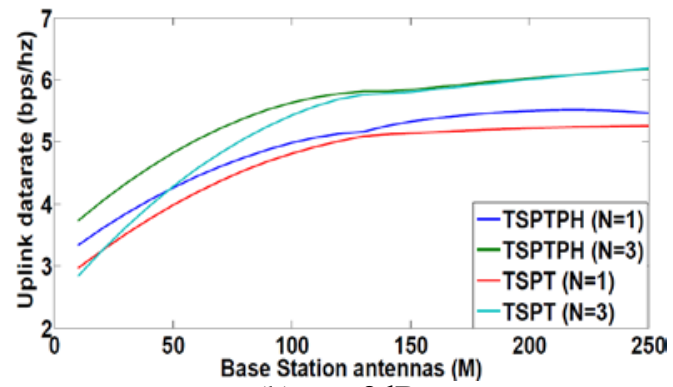

(b) $\sigma=8 \mathrm{~dB}$

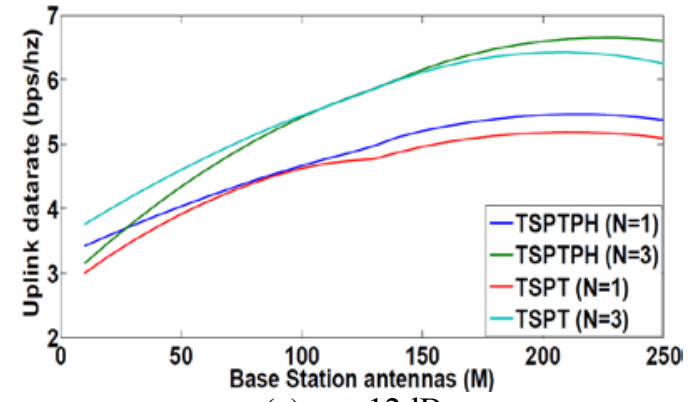

(c) $\sigma=12 \mathrm{~dB}$

Uplink data-rate (40 hops)

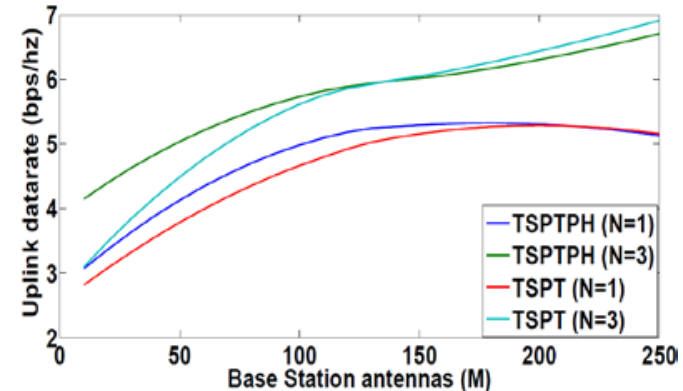

(a) $\sigma=0 \mathrm{~dB}$

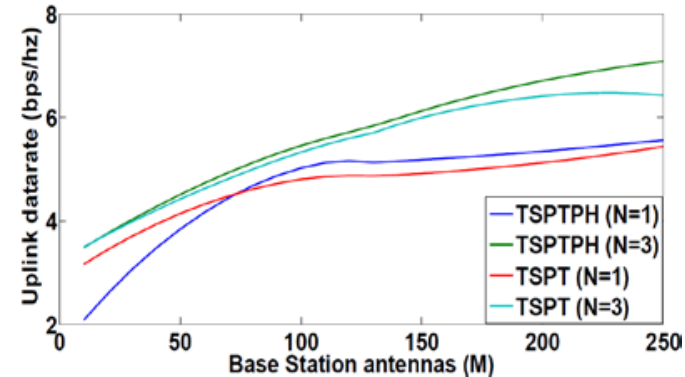

(b) $\sigma=8 \mathrm{~dB}$

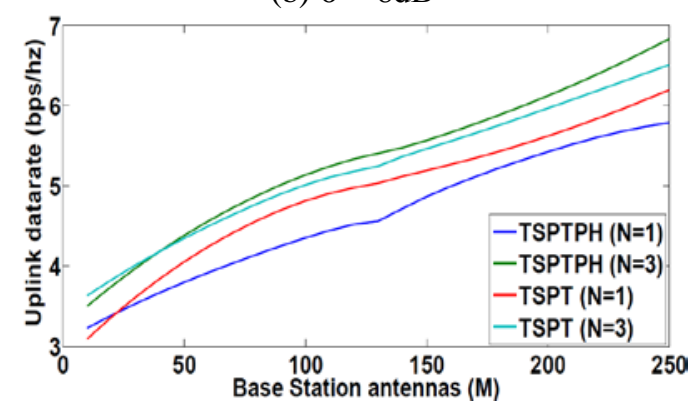

(c) $\sigma=12 \mathrm{~dB}$

Uplink data-rate (30 hops)

Fig. 6. Uplink data-rate with respect to antennas for $\mathrm{K}=15$ and 40 hops and 30 hops

\section{Conclusion}

The efficiency of multi-cell MMIMO system is severely restricted by pilot contamination. In the TSPTPH, user hops to a new pilot signal in each transmission slot. Therefore, a group of users contaminating the channel is changing, thereby changing channel coefficient $\beta$ which is function of lognormal distribution $z$ and radial distance $r$ in each transmission slot. This leads to reduction in the channel estimation error and improves uplink performance. It is noticed that the performance of TSPTPH for all three path-loss model is significantly better for reuse factor $N=3$, for both $K=8$ and $K=15$ with 40 hops. TSPTPH shows average improvement in uplink performance of $4 \%$ for $K=8$ and $2.5 \%$ for $K=15$ with 40 hops whereas with 30 hops improvement is $1.5 \%$ for $K=8$ and $2 \%$ for $K=15$. For $N=1$, performance of TSPTPH and TSPT nearly same. From the results it is concluded that proposed system works well in any type of radio environment. 


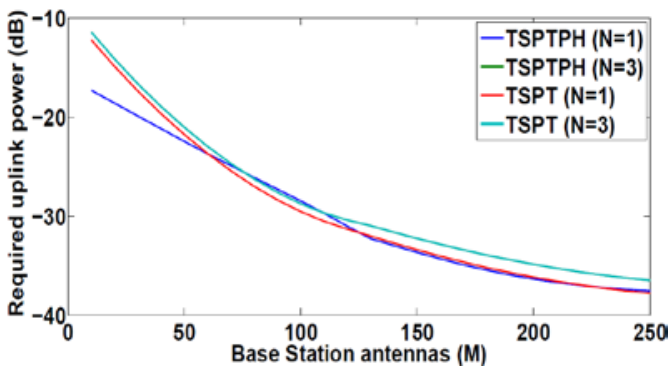

(a) $\sigma=0 \mathrm{~dB}$

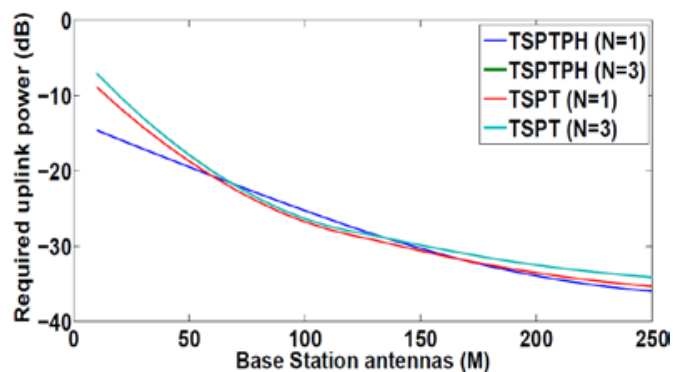

(b) $\sigma=8 \mathrm{~dB}$

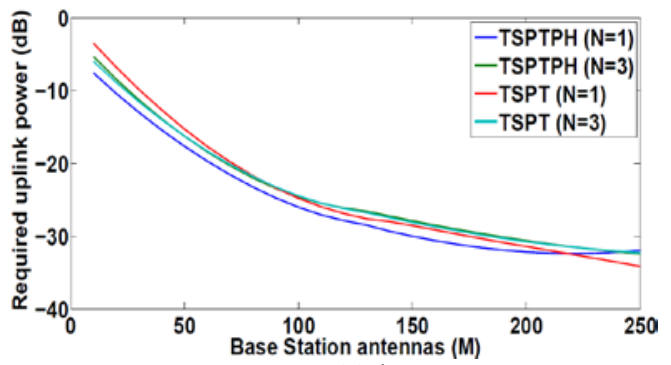

(c) $\sigma=12 \mathrm{~dB}$

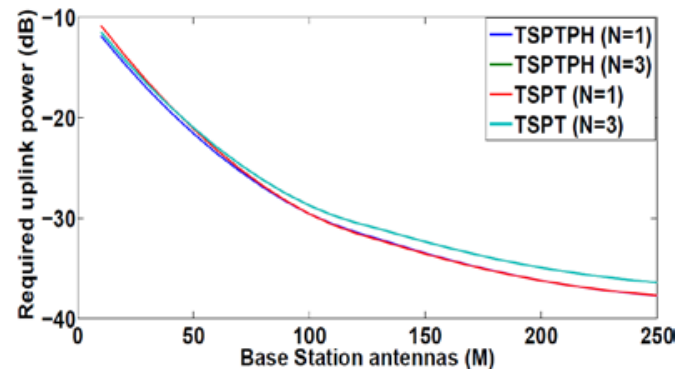

(a) $\sigma=0 \mathrm{~dB}$

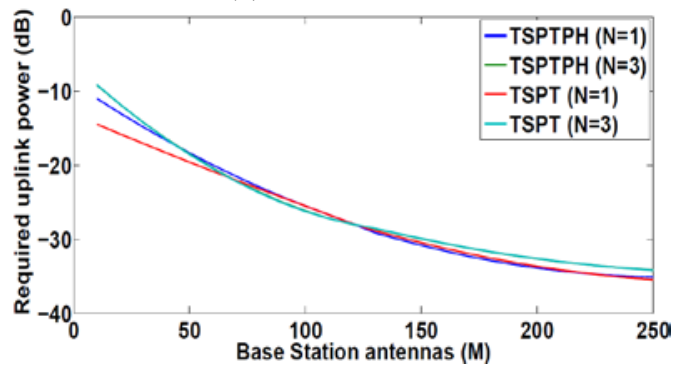

(b) $\sigma=8 \mathrm{~dB}$

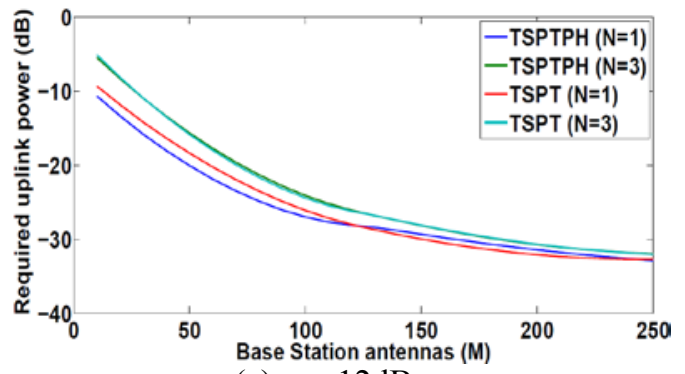

(c) $\sigma=12 \mathrm{~dB}$

Required uplink SNR for $\mathrm{K}=15,30$ hops

Required uplink SNR for K=15, 40 hops

Fig. 7. Required uplink SNR for $\mathrm{K}=15,40$ hops and 30 hops

In addition to this, uplink SNR required for data transmission is also calculated for spectral efficiency of $1 \mathrm{bit} / \mathrm{sec} / \mathrm{Hz}$. Observed results show that, as the number of BS antennas increases, uplink SNR required for data transmission decreases. It is also observed that uplink SNR increases as the shadowing effect increases for both the systems. Thus, the performance of proposed TSPTPH system enhances uplink SINR, achievable data-rate and simultaneously reduces the power consumption by reducing the uplink SNR required for data transmission at UE end.

\section{Appendix A}

Seven cell structure is considered each with radius $R$ and each cell is having six interfering cells as shown in Fig. 1.

$$
R_{1}=R_{2}=R_{3}=R_{4}=R_{5}=R_{6}=R_{7}=R
$$

SINR and data-rate depend on $\beta$, which is the function of $z$ and and radial distance $r$ between UE and BS. Therefore 


$$
\begin{gathered}
\operatorname{SINR}=g\left(R_{1} R_{2} \cdots R_{6}\right) \\
E(\operatorname{SINR})=\int_{0}^{R_{1}} \int_{0}^{R_{2}} \cdots \int_{0}^{R_{6}} f\left(R_{1}, R_{2} \cdots R_{6}\right) d R_{1} d R_{2} \cdots d R_{6}
\end{gathered}
$$

where, $f\left(R_{1}, R_{2} \cdots R_{6}\right)$ is density function. Interference between two cells will be zero when pilot signals are not reuse i.e.

$$
f\left(R_{1}, R_{2} \cdots R_{6}\right)=f\left(R_{1}\right) f\left(R_{2}\right) \cdots f\left(R_{6}\right)
$$

If pilot signals are reused in all the neighbouring cells, at any point user position $\left(x_{1}, y_{1}\right)$ with respect to cell having radius $R_{1}$ with centre $\left(\alpha_{1}, \gamma_{1}\right)$ will have probability density function of

$$
\begin{gathered}
f\left(R_{1}\right)=f_{x y}\left(x_{1}, y_{1}\right) \\
f\left(R_{1}\right)=\left\{\begin{array}{cc}
\frac{1}{\pi R_{1}^{2}}\left[\left(x_{1}-\alpha_{1}\right)^{2}+\left(y_{1}-\gamma_{1}\right)^{2}\right] & \text { if } \text { UE position } \leq R_{1}^{2} \\
0 & \text { otherwise }
\end{array}\right.
\end{gathered}
$$

Similarly

$$
\begin{gathered}
f\left(R_{2}\right)=f_{x y}\left(x_{2}, y_{2}\right) \\
\left\{\begin{array}{cc}
\frac{1}{\pi R_{2}^{2}}\left[\left(x_{2}-\alpha_{2}\right)^{2}+\left(y_{2}-\gamma_{2}\right)^{2}\right] & \text { if } U E \text { position } \leq R_{2}^{2} \\
0 & \text { otherwise } \\
\therefore f_{R_{1}}\left(x_{1}\right)=\int f_{R_{1}}\left(x_{1}, y_{1}\right) d y_{1}
\end{array}\right.
\end{gathered}
$$

Where limits are

$$
\begin{gathered}
\gamma_{1}-\sqrt{R_{1}^{2}-\left(x_{1}-\alpha_{1}\right)^{2}} \leq y_{1} \leq \gamma_{1}+\sqrt{R_{1}^{2}+\left(x_{1}-\alpha_{1}\right)^{2}} \\
f_{R_{1}}\left(x_{1}\right)=\int_{\gamma_{1}-\sqrt{R_{1}^{2}-\left(x_{1}-\alpha_{1}\right)^{2}}}^{\gamma_{1}+\sqrt{R_{1}^{2}+\left(x_{1}-\alpha_{1}\right)^{2}}} \frac{1}{\pi R_{1}^{2}} d y \\
f_{R_{1}}\left(x_{1}\right)=\frac{1}{\pi R_{1}^{2}} 2 \sqrt{R_{1}^{2}-\left(x_{1}-\alpha_{1}\right)^{2}} \quad \text { where, } \alpha_{1}-R_{1} \leq x_{1} \leq \alpha_{1}+R_{1}
\end{gathered}
$$

Now considering all six interfering cells users, $E(\operatorname{SINR})$ is given as

$$
E(\operatorname{SINR})=\int_{\alpha_{1}-R_{1}}^{\alpha_{1}+R_{1}} \cdots \int_{\alpha_{6}-R_{6}}^{\alpha_{6}+R_{6}} g\left(R_{1}, \cdots R_{6}\right) \times\left(\frac{2}{\pi}\right)^{6} \times \prod_{m=1}^{6} \frac{\sqrt{R_{m}{ }^{2}-\left(x_{m}-\alpha_{m}\right)^{2}}}{R_{m}{ }^{2}} d x_{1} \cdots d x_{6} \text { (33) }
$$




\section{References}

[1] F. Rusek, D. Persson, and B. K. Lau, "Scaling up MIMO: Opportunities and challenges with very large arrays,” IEEE Signal Processing Magazine, vol. 30, no. 1, pp. 40-60, 2013. Article (CrossRef Link)

[2] L. Lu, G. Li, A. Swindlehurst, A. Ashikhmin, and R. Zhang, "An overview of Massive MIMO: Benefits and challenges,” IEEE Journal of Selected Topics in Signal Processing, vol. 8, no. 5, pp. 742-758, Oct 2014. Article (CrossRef Link)

[3] E. Larsson, O. Edfors, F. Tufvesson, and T. Marzetta, "Massive MIMO for next generation wireless systems,” IEEE Communications Magazine, vol. 52, pp. 186-195, 2014.

Article (CrossRef Link)

[4] D. C. Araújo and T. Maksymyuk and A. L. F. de Almeida and T. Maciel and J. C. M. Mota and M. Jo "Massive MIMO: survey and future research topics," IET Communications, vol. 10, pp. 1938-1946, 2016. Article (CrossRef Link)

[5] T. Marzetta, "Noncooperative cellular wireless with unlimited numbers of base station antennas,” IEEE Transactions on Wireless Communications, vol. 9, no. 11, pp. 3590-3600, Nov. 2010. Article (CrossRef Link)

[6] V. Saxena, G. Fodor, and E. Karipidis, "Mitigating pilot contamination by pilot reuse and power control schemes for Massive MIMO systems,” in Proc. of Vehicular Technology Conference, vol. 81, pp. 1-6, May 2015. Article (CrossRef Link)

[7] X. Zhu, Z. Wang, L. Dai, and C. Qian, "Smart pilot assignment for massive MIMO," IEEE Communications Letters, vol. 19, no. 9,pp. 1644-1647, September 2015. Article (CrossRef Link)

[8] J. Jose, A. Ashikhmin, T. Marzetta, and S. Vishwanath, "Pilot contamination problem in multi-cell TDD systems," in Proc. of IEEE International Symposium on Information Theory, vol. 10, pp. 2184-2188, 2009. Article (CrossRef Link)

[9] J. Jose, A. E. Ashikhmin, T. L. Marzetta, and S. Vishwanath, "Pilot contamination and precoding problem in multi-cell TDD systems," IEEE Transactions on Wireless Communications, vol. 10, no. 8, pp. 2640-2651, 2011. Article (CrossRef Link)

[10] H. Jakob, ten Brink Stephan, and D. Merouane, "Comparison of linear precoding schemes for downlink massive MIMO,” in Proc. of IEEE ICC, Ottawa, Canada, pp. 2135-2139, 2012. Article (CrossRef Link)

[11] A. Ruperee and S. Nema, "Fractional frequency reuse to enhance down link performance in MU Massive MIMO," in Proc. of IEEE International Conference on Wireless Communications, Signal Processing and Networking, pp. 1804-1809, March 2016. Article (CrossRef Link)

[12] X. Ge, R. Zi, H. Wang, J. Zhang, M. Jo, "Multi-User Massive MIMO Communication Systems Based on Irregular Antenna Arrays," IEEE Transactions on Wireless Communications, vol. 15, no. 8, pp. 5287-5301, 2016. Article (CrossRef Link)

[13] K. Appaiah, A. Ashikhmin, and T. L. Marzetta, "Pilot contamination reduction in multi-user TDD systems," in Proc. of IEEE International Conference on Communications, pp. 1-5, May 2010. Article (CrossRef Link)

[14] T. Yao and Y. Li, "Pilot contamination reduction by shifted frame structure in Massive MIMO TDD wireless system," Wuhan University Journal of Natural Sciences, vol. 20, no. 3, pp. 221-228, 2015. Article (CrossRef Link)

[15] S. Jin, X. Wang, Z. Li, K.-K. Wong, and Y. Huang, "On Massive MIMO zero-forcing transceiver using time-shifted pilots,” IEEE Transactions on Vehicular Technology, vol. 65, no. 1, pp. 59-74, January 2016. Article (CrossRef Link)

[16] W. A. W. M. Mahyiddin, P. A. Martin, and P. J. Smith, "Performance of synchronized and unsynchronized pilots in finite Massive MIMO systems," IEEE Transactions on Wireless Communications, vol. 14, no. 12, pp. 6763-6776, Dec 2015. Article (CrossRef Link) 
[17] V. D. Nguyen, H. V. Nguyen, and O. S. Shin, "Channel estimation based on time-shifted pilots in multicell Massive MIMO systems," in Proc. of International. Conference on Communications, Management and Telecommunications, pp. 83-87, Dec 2015.

Article (CrossRef Link)

[18] F. Fernandes, A. Ashikhmin, and T. L. Marzetta, "Inter-cell interference in noncooperative TDD large scale antenna systems," IEEE Journal on Selected Areas in Communications, vol. 31, no. 2, pp. 192-201, February 2013. Article (CrossRef Link)

[19] J. H. Sorensen and E. de Carvalho, "Pilot decontamination through pilot sequence hopping in massive MIMO systems," in Proc. of IEEE Global Communication Conference, pp. 3285-3290, Dec 2014. Article (CrossRef Link)

[20] A. Ruperee and S. Nema, "Asynchronous pilot transmission with pilot sequence hopping for improved channel estimation in massive MIMO system," in Proc. of the IEEE International Conference on Recent Innovations in Signal processing and Embedded Systems, pp. 107112, Oct 2017. Article (CrossRef Link)

[21] D. C. Cox, R. R. Murray, and A. W. Norris, "800-mHz attenuation measured in and around suburban houses,” Bell Lab. Technical Journal, vol. 63, no. 6, pp. 921-954, July 1984. Article (CrossRef Link)

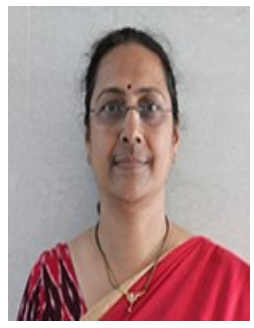

Amrita Ruperee received Bachelor of Engineering in Electronics in 1991 from Govt. College of Engineering, Ujjain, India and M.Tech. in Digital communication in 2000 from MANIT, Bhopal, India. Currently pursuing PhD in the area of Wireless Communication in S. N. D. T. University, Mumbai, India. With more than 20 years of teaching experience, she is currently working as Associate Professor in department of Electronics and Telecommunication Engineering at Vidyavardhini's College of Engineering and Technology, Mumbai, India. Her research areas include Mobile Communication, Wireless Communication

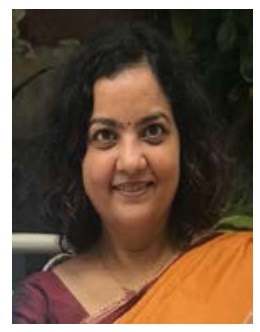

Shikha Nema received Bachelor of Engineering in Electronics in 1994, M.Tech. in Digital communication in 2004 and Ph.D. in 2010 from MANIT, Bhopal, MP, India. With more than 16 years of teaching and 4 years of industrial experience, she is currently working as Professor \& Head, Electronics \& Communication Engineering with S. N. D. T. University, Mumbai, India. Her research areas include Mobile Communication, Wireless Networks and Optical Communication. She has more than 50 publications to her credit at national and international level in reputed conferences and journals. 